Theory-based scaling of the SOL width in circular limited tokamak plasmas

This content has been downloaded from IOPscience. Please scroll down to see the full text.

View the table of contents for this issue, or go to the journal homepage for more

Download details:

This content was downloaded by: riccipaolo

IP Address: 128.179.255.118

This content was downloaded on 13/08/2015 at 12:13

Please note that terms and conditions apply. 


\title{
LETTER
}

\section{Theory-based scaling of the SOL width in circular limited tokamak plasmas}

\author{
F.D. Halpern ${ }^{1}$, P. Ricci ${ }^{1}$, B. Labit ${ }^{1}$, I. Furno ${ }^{1}$, S. Jolliet ${ }^{1}$, J. Loizu ${ }^{1}$, \\ A. Mosetto ${ }^{1}$, G. Arnoux ${ }^{2,3}$, J.P. Gunn ${ }^{4}$, J. Horacek ${ }^{5}$, M. Kočan ${ }^{6}$, \\ B. LaBombard ${ }^{7}$, C. Silva ${ }^{2,8}$ and JET-EFDA Contributors ${ }^{\mathrm{a}}$ \\ ${ }^{1}$ École Polytechnique Fédérale de Lausanne (EPFL), Centre de Recherches en Physique des \\ Plasmas, Association Euratom-Confédération Suisse, CH-1015 Lausanne, Switzerland \\ 2 JET-EFDA, Culham Science Centre, OX14 3DB, Abingdon, UK \\ ${ }^{3}$ EURATOM/CCFE Fusion Association, Culham Science Centre, Abingdon, Oxon, OX14 \\ 3DB, UK \\ ${ }^{4}$ CEA, IRFM, 13108 Saint-Paul-lez-Durance, France \\ ${ }^{5}$ Institute of Plasma Physics AS CR, v.v.i., Association EURATOM/IPP.CR, Za Slovankou \\ 1782/3, 182 00, Praha 8, Czech Republic \\ ${ }^{6}$ ITER Organization, Route de Vinon-sur-Verdon, 13115 Saint-Paul-lez-Durance, France \\ ${ }^{7}$ Massachusetts Institute of Technology, Cambridge, MA 02139, USA \\ ${ }^{8}$ Associação Euratom/IST, Instituto de Plasmas e Fusão Nuclear, Instituto Superior Técnico, \\ Universidade de Lisboa, Portugal
}

Received 2 July 2013, accepted for publication 17 October 2013

Published 4 November 2013

Online at stacks.iop.org/NF/53/122001

\begin{abstract}
A theory-based scaling for the characteristic length of a circular, limited tokamak scrape-off layer (SOL) is obtained by considering the balance between parallel losses and non-linearly saturated resistive ballooning mode turbulence driving anomalous perpendicular transport. The SOL size increases with plasma size, resistivity, and safety factor $q$. The scaling is verified against flux-driven non-linear turbulence simulations, which reveal good agreement within a wide range of dimensionless parameters, including parameters closely matching the TCV tokamak. An initial comparison of the theory against experimental data from several tokamaks also yields good agreement.
\end{abstract}

(Some figures may appear in colour only in the online journal)

The peak heat load onto the plasma facing components of tokamak devices depends on the scrape-off layer (SOL) width [1-9], which results from a balance between plasma injection from the core region, turbulent transport, and losses to the divertor or limiter. While determining the SOL width and understanding the transport mechanism involved are critical issues for ITER and all future tokamak devices, so far there are no first-principle methods from the theory to predict the SOL width even for the simplest circular, limited plasmas.

The present letter reports on a theory-based scaling of the SOL characteristic pressure radial scale length $L_{p}=-p / \nabla p$ at the outboard mid-plane of an inboard limited plasma. The SOL width results from a power balance between parallel losses and anomalous transport due to low frequency interchange turbulence. Our work concentrates on a relatively simple, circular, inner-wall limited configuration.

a See the appendix of Romanelli F. et al Proc. 23rd IAEA Fusion Energy Conf. 2010 (Daejeon, Korea).
Understanding a circular configuration is a departure point for studying more complicated geometries or regimes, and is important for the start-up phase of ITER. The scaling derived in the present letter is verified against global, flux driven, 3D turbulence simulations of the SOL and agrees reasonably well with experimental data from inner-wall limited tokamak discharges. We note that, in the past, there have been attempts to explain the SOL width using 2D models (see e.g., [10-13]).

Recent studies using an electromagnetic drift-reduced Braginskii fluid model [14] have shown that the magnitude of the turbulent fluxes in the SOL can be predicted using the non-linear local flattening of the pressure profile caused by the linear modes $[15,16]$, the so-called gradient removal mechanism. Here, we summarize the results of this saturation model, which is fully derived in [15]. In non-linear, flux-driven simulations, it is observed that turbulent saturation occurs when the pressure non-linearity exhausts the linear drive from the background gradient, i.e. $p_{1} / p_{0} \sim \sigma_{r} / L_{p}$. 
(The subscripts 0 and 1 indicate background and perturbed quantities, respectively and $\sigma_{r}$ is radial extension of the mode.) In the regime of interest here, $k_{\theta} L_{p}>1\left(k_{\theta}\right.$ is the poloidal wavenumber), using a non-local linear theory, it can be shown that $\sigma_{r} \approx \sqrt{L_{p} / k_{\theta}}[17,18]$. Note that $\sigma_{r}$ is determined by combining turbulent $\left(k_{\theta}^{-1}\right)$ and equilibrium $\left(L_{p}\right)$ spatial scales, consequently, our model specifically deals with the turbulent saturation of meso-scale structures such as the ones inferred from experimental measurements. We also note that $\sigma_{r}$ is not affected by magnetic shear, in the typical parameter range of limited discharges [17, 19]. Using the leading order contribution of the continuity equation, $\partial_{\mathrm{t}} p+\nabla_{\perp} \cdot\left(\boldsymbol{v}_{\boldsymbol{E} \times \boldsymbol{B}} p\right)=0$, it is possible to relate the pressure and electrostatic potential fluctuations assuming that $\boldsymbol{E} \times \boldsymbol{B}$ shear flow is negligible, i.e. $\gamma p_{1} / p_{0} \sim k_{\theta} \phi_{1} /\left(B L_{p}\right)$, where $\gamma$ is the linear growth rate of the instability that dominates the non-linear dynamics. Therefore, the time-averaged radial $\boldsymbol{E} \times \boldsymbol{B}$ turbulent flux, $\Gamma=\left\langle k_{\theta} p_{1} \phi_{1}\right\rangle_{\mathrm{t}} / B$, can be estimated as $\Gamma \sim p_{0} \gamma / k_{\theta}$. The balance between perpendicular turbulent transport, $\partial_{r} \Gamma \sim \Gamma / L_{p} \sim p_{0} \gamma /\left(k_{\theta} L_{p}\right)$, and the parallel losses at the sheath, $\nabla_{\|}\left(p v_{\| \mathrm{e}}\right) \sim p_{0} c_{\mathrm{s}} /(q R)$, leads to an estimate of the profile length

$$
L_{p} \sim\left(q R / c_{\mathrm{s}}\right)\left(\gamma / k_{\theta}\right)_{\max },
$$

where $R$ is the tokamak major radius, $q \approx(r / R) B_{\phi} / B_{\theta}$ is the cylindrical tokamak safety factor, and $c_{\mathrm{s}}=\sqrt{\left(T_{\mathrm{i}}+T_{\mathrm{e}}\right) / m_{\mathrm{i}}}$ is the sound speed. In deriving equation (1), it is assumed that the flux is driven by a single mode that leads to the flattest possible pressure profile. Furthermore, it is assumed that parallel temperature gradients are weak. This assumption breaks down in high-density low-temperature discharges, where parallel temperature gradients develop and the effect of neutral particles becomes more important [20].

The pressure gradient length can now be computed assuming that SOL turbulence is driven by resistive ballooning modes (RBMs). Non-linear, 3D electromagnetic simulations have addressed the nature of SOL turbulence in limited circular plasmas, finding that RBMs dominate the plasma dynamics $[16,21]$. The SOL is very collisional compared to the plasma core, and, in the absence of magnetic field line periodicity, RBMs are dominant over non-linearly driven drift waves [21]; at the same time linearly unstable drift waves are efficiently damped by the magnetic shear [19]. Electromagnetic induction has a destabilizing effect on RBMs near the ideal ballooning threshold [16]. Ion temperature effects could introduce the ion temperature gradient mode (ITG), but ITGs are important at much lower collisionalities than in the regime of interest $\left(v_{\star} \sim\right.$ 10) [22]. It is also possible to estimate the non-linear regime instability using linear calculations and an estimate of the radial flux driven by each instability [23]. This calculation, when carried out for experimentally relevant parameters (connection length, collisionality, plasma size, positive magnetic shear, etc), confirms the importance of RBMs in the SOL.

In order to obtain $L_{p}$ through equation (1), we now seek for the typical growth rate and poloidal wave length of RBMs. A simple dispersion relation for RBMs, valid in the low $\beta$ limit and considering only the low-field side mid-plane region (where the curvature drive for the modes is strong), can be obtained from the reduced resistive magnetohydrodynamic (MHD) equations:

$$
\left(\gamma^{2}-\gamma_{\mathrm{b}}^{2}\right) k_{\theta}^{2}=-\gamma \frac{\mu_{0} \sigma_{\|} v_{\mathrm{a}}^{2}}{q^{2} R^{2}}(1-\alpha)-\gamma^{3} \mu_{0} \sigma_{\|} .
$$

Here $\gamma_{\mathrm{b}}=c_{\mathrm{s}} \sqrt{2 /\left(R L_{p}\right)}$ is the typical RBM growth rate, $\sigma_{\|}$ is the parallel Spitzer conductivity (neoclassical corrections to the Spitzer formula are neglected, since the large $v_{\star}$ in the SOL prevents trapping), $v_{\mathrm{a}}=B / \sqrt{\mu_{0} m_{\mathrm{i}} n}$ is the Alfvén speed, $\alpha=q^{2} \beta R / L_{p}$ is the ideal ballooning stability parameter, and we have approximated $\nabla_{\|} \sim 1 /(q R)$.

The term on the right hand side of equation (2) results from the parallel dynamics in the Ohm's law. Parallel streaming is mediated by an effective conductivity $\sigma_{\|}(1-\alpha)$ that allows RBMs to grow at lower $k_{\theta}$ provided that (a) the conductivity decreases, (b) $\beta$ increases or (c) the connection length $L_{\mathrm{c}} \sim q R$ increases [16]. The poloidal wavelength of the mode is a key component of the saturation rule given by equation (1). By estimating the strength of the parallel damping effect, equation (2) leads to $k_{\theta}^{2}=\left(\mu_{0} \sigma_{\|} v_{\mathrm{a}}^{2}\right)(1-\alpha) /\left(q^{2} R^{2} \gamma_{\mathrm{b}}\right)=k_{\mathrm{b}}^{2}$.

The SOL pressure gradient length $L_{p}$ can now be obtained from equation (1) together with the RBM estimates $k_{\theta}=k_{\mathrm{b}}$ and $\gamma=\gamma_{\mathrm{b}}$. In order to normalize equation (1), we introduce a characteristic length $L_{\perp}=\rho_{\mathrm{s}}=$ $c_{\mathrm{s}} / \omega_{\mathrm{ci}}=\sqrt{\left(T_{\mathrm{i}}+T_{\mathrm{e}}\right) m_{\mathrm{i}}} /(e B)$ and the adimensionalized Spitzer resistivity $v=\left(e^{2} n R\right) /\left(m_{\mathrm{i}} \sigma_{\|} c_{\mathrm{s}}\right)$. The scaling obtained is

$$
\tilde{L}_{p} \tilde{R}^{-1 / 2}=\left[2 \pi \alpha_{\mathrm{d}}(1-\alpha)^{1 / 2} / q\right]^{-1 / 2},
$$

where $L_{p}$ and $R$ are normalized to $\rho_{\mathrm{s}}$ (denoted by a tilde) and $\alpha_{\mathrm{d}}=2^{-3 / 4} v^{-1 / 2}\left(R / L_{p}\right)^{1 / 4} /(2 \pi q)$ is a dimensionless variable that measures the strength of the parallel damping term in equation (2) [24, 25].

Equation (3) is particularly amenable for comparisons with flux-driven non-linear turbulence simulations. To that effect, we have carried out a verification of the scaling using global, non-linear simulations of the SOL dynamics using the GBS turbulence code, a numerical implementation of a global drift-reduced Braginskii model. The physical model and the code are fully described in [14]. Starting from the Braginskii fluid equations [26], we adopt the orderings $\mathrm{d} / \mathrm{d} t \ll \omega_{\mathrm{ci}}$, $k_{\perp} \gg k_{\|}$, and $T_{\mathrm{i}} \ll T_{\mathrm{e}}$, which result in drift-reduced electromagnetic equations describing the non-linear evolution of the plasma density, vorticity, electron and ion parallel velocity, perturbed poloidal magnetic flux, and electron temperature. The generalized Ohm's law includes plasma conductivity, electron inertia, electromagnetic fluctuations, and diamagnetic stabilizations. The equations are a two-fluid superset of the reduced MHD equations and they can describe the resistive and inertial branches of drift waves and ballooning modes, and, in addition, the ideal ballooning mode.

The plasma dynamics is evolved within an annulus in the open magnetic field line region of the plasma vessel. Entire flux surfaces, up to the limiter, are included in the simulation domain. We use a simple circular geometry with a toroidal limiter on the high field side mid-plane, with constant $q$ and constant magnetic shear $\hat{s}=r q^{\prime} / q$. Boundary conditions at the magnetic pre-sheath entrance, where the plasma interfaces with the limiter plates and the ion drift approximation breaks down, are computed following the model by Loizu et al [27]. 
The drift-reduced Braginskii model used for the simulations does not separate fluctuations from the background profiles, and no separation between equilibrium and turbulent length scales is imposed a priori. The plasma density, temperature, potential, and parallel velocities are initialized using smooth profiles with a small perturbation superimposed. Plasma sources, which mimic the plasma outflow from the core, are then introduced. The pressure gradient increases until linearly unstable modes appear, driving turbulence that leads to perpendicular transport. Over a longer period, the modes saturate and a non-linear turbulent steady state is achieved. Since interchange turbulence and sheared flows occur in the simulated plasma, blob dynamics are also present in the simulations. The plasma gradients are naturally reached as a balance between plasma injection, turbulent transport, and parallel losses at the plasma sheaths. Therefore, the GBS code is especially suited for verifying the dimensionless scaling given by equation (3).

An extensive simulation campaign, the details of which will be published in a longer paper, was carried out to investigate the inner-wall limited SOL parameter space. In essence, we are interested in simulating limited plasmas where RBMs dominate transport and to maximize the range of the dimensionless parameter space probed. The resistivity and $q$ are varied to investigate the effect of the parallel dynamics of the turbulent modes. The plasma $\beta$ is increased by several orders of magnitude to test the strength of electromagnetic effects predicted by equation (2). Crucially, the normalized major radius $\tilde{R}$ is increased by a factor of 4 in order to test the effects of the plasma size predicted by the scaling. The simulations lie within the following range of parameters: $q=3,4,6 ; \beta=0$ to $10^{-3} ; v=0.01,0.1,1 ; \tilde{R}=500$, 1000,$2000 ; \hat{s}=0,1,2$. The poloidal resolution of the simulations is such that turbulent structures with $k_{\theta} \rho_{s}$ up to 2 are included. Taking TCV as an example $(R=0.88 \mathrm{~m}$, $B=1.4 \mathrm{~T}, T_{\mathrm{e}, \mathrm{SOL}} \approx 15 \mathrm{eV}, n_{\mathrm{e}, \mathrm{SOL}} \approx 3 \times 10^{18} \mathrm{~m}^{-3}$ ) we obtain $\tilde{R} \approx 2000, v \approx 0.01, \alpha_{\mathrm{d}} \approx 0.5, \beta \approx 10^{-5}, v_{\star} \approx 40$, while $q=3-6$ and $\hat{s} \approx 2$. The SOL pressure gradient length typically has a width of a few centimetres, $\tilde{L}_{p} \sim 100$, $\tilde{R} / \tilde{L}_{p} \approx 20$. Our simulations are able to match this regime closely - a set of simulations with parameters equivalent to the TCV SOL were carried out.

We now verify the dimensionless scaling. The equilibrium pressure profiles observed in the turbulent steady state typically have the form $p \sim \exp \left(-\tilde{r} / \tilde{L}_{p}\right)$ with $\tilde{L}_{p}$ ranging from 25 to 150 . The ideal stability parameter $\alpha$ ranges from 0 to 0.25 , while $\alpha_{\mathrm{d}}$ (which only depends weakly on $\tilde{R} / \tilde{L}_{p}$ ) ranges between 0.01 and 0.6. From $\tilde{L}_{p}, \alpha$, and $\alpha_{\mathrm{d}}$ we obtain the left and right hand sides of equation (3), which are shown as a scatter plot in figure 1 . Note that, by splitting the left and right hand sides, the plasma size dependence and the dependence on $q$ and $v$ can be observed separately. It is concluded that the dependence on plasma size (through $\tilde{R}$ ) and the dependence on the connection length $\sim q^{-1}$ are both well described. Overall, good agreement is found between the non-linear simulation results and the prediction of the analytical theory over the entire parameter range. This is due to the fact that the non-linear turbulent spectra observed in GBS simulations are typically dominated by a few resonant long wavelength modes with $k_{\theta} \rho_{\mathrm{s}} \sim 0.1$. Furthermore, secondary instabilities, such as

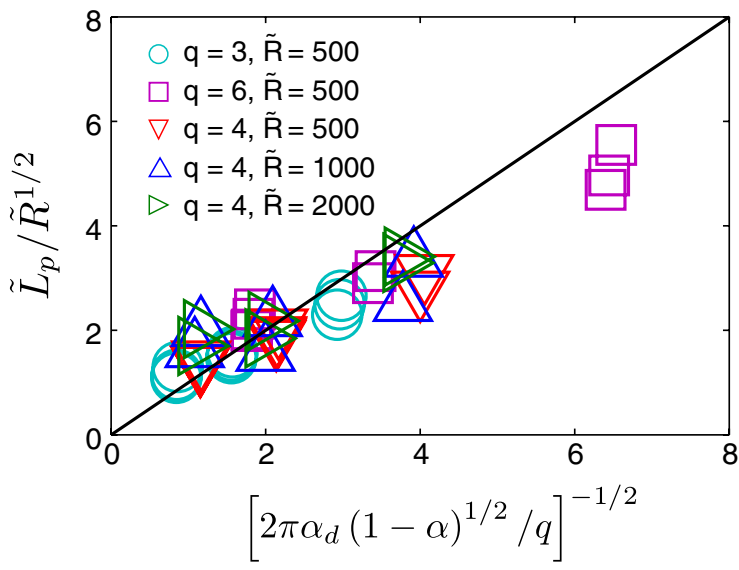

Figure 1. Dimensionless pressure gradient length $\tilde{L}_{p}$ normalized by $\tilde{R}^{1 / 2}$ is shown as a function of the dimensionless parameters $\alpha, \alpha_{\mathrm{d}}$, and $q$. All the parameters (except for $q$, which is imposed) are self consistently obtained in the GBS simulations. The solid line represents equation (3).

the Kelvin-Helmholtz mode, do not to play a significant role saturating SOL turbulence in the regime of interest here [15].

Starting from equation (1) and using $\gamma=\gamma_{\mathrm{b}}$ and $k_{\theta}=k_{\mathrm{b}}$, it is also possible to obtain a scaling in terms of engineering parameters. This is a more practical approach for comparing against experimental data and to extrapolate $L_{p}$ to future devices. Since $\beta$ is very small in the SOL of circular limited plasmas, we take the electrostatic limit $\alpha \ll 1$, which leads to the following scaling:

$L_{p} \approx 7.22 \times 10^{-8} q^{8 / 7} R^{5 / 7} B_{\phi}^{-4 / 7} T_{\mathrm{e} 0}^{-2 / 7} n_{\mathrm{e} 0}^{2 / 7}\left(1+\frac{T_{\mathrm{i} 0}}{T_{\mathrm{e} 0}}\right)^{1 / 7}$

Here $q, n_{0}, T_{\mathrm{e} 0}$, and $T_{\mathrm{i} 0}$ must be provided at the low-field-side midplane of the last closed flux surface (LCFS), while $R$ and $B_{\phi}$ must be provided at the magnetic axis. All quantities are expressed using SI units except for $T_{\mathrm{e} 0}$ and $T_{\mathrm{i} 0}$, which are given in $\mathrm{eV}$. The constant factor arises from the Spitzer conductivity, and we have approximated the Coulomb logarithm $\ln \Lambda \approx 15$. The factor of $q^{8 / 7} \sim I_{p}^{-8 / 7}$ results in a strong dependence of $L_{p}$ on the plasma current, while the explicit dependence on $T_{\mathrm{e}}$, $T_{\mathrm{i}}$, and $n$ is rather weak.

Equation (4) has been compared against experimental data from inner-wall limited discharges in five devices, as shown in figure 2. The abscissa provides experimental measurements while the ordinate provides theoretical predictions given by equation (4). The data used to compute figure 2 are shown in table 1 . Note that $\alpha \approx 0.01 \ll 1$ in the SOL of these discharges, justifying our neglect of electromagnetic effects in equation (4). The data involve inner-wall limited discharges from Alcator C-Mod [1], COMPASS, JET [8,9], $\mathrm{TCV}$, and Tore Supra [2,7]. The data used in figure 2 are shown in table 1 . The profile data were measured using reciprocating Langmuir probes, except for Tore Supra, where a retarding field analyser was used to measure $T_{\mathrm{i}}$ and a tunnel probe was used to measure $n$ and $T_{\mathrm{e}} . L_{p}$ has been obtained by determining the characteristic length of an exponentially decaying pressure profile. The horizontal bars indicates the 


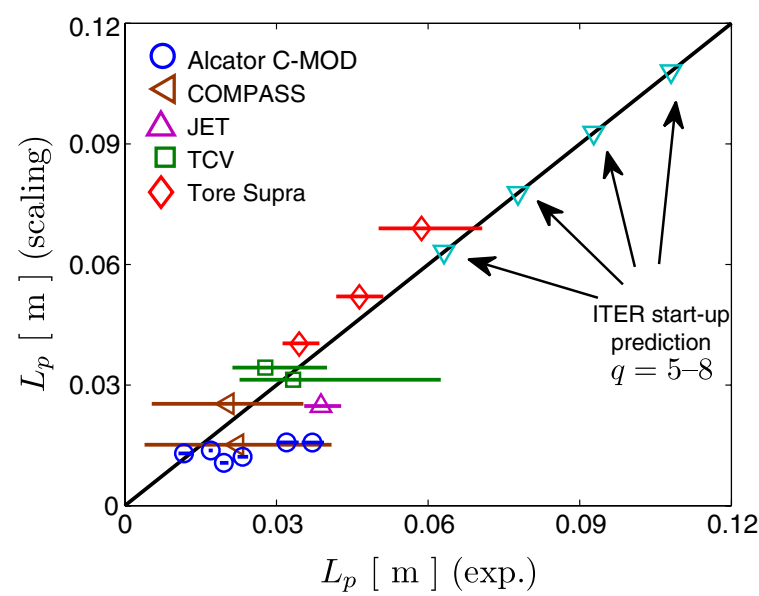

Figure 2. Scaling given by equation (4) is compared against experimental data from inner-wall limited discharges. The abscissa and ordinate provide, respectively, the experimental data and the prediction of the theoretical scaling. The horizontal bars indicate the 95\% confidence interval obtained from the fitting of $L_{p}$. ITER start-up predictions are displayed as 'down-triangles'.

Table 1. Plasma parameters used to obtain figure 2. The units used are as follows: $R(\mathrm{~m}), B_{\phi}(\mathrm{T}), T_{\mathrm{e}}(\mathrm{eV}), n_{\mathrm{e}}\left(10^{19} \mathrm{~m}^{-3}\right), L_{p}(\mathrm{~cm})$.

\begin{tabular}{llllllll}
\hline Case & $q$ & $R$ & $B_{\phi}$ & $T_{\mathrm{e}}$ & $n_{\mathrm{e}}$ & $L_{p}^{\exp }$ & $L_{p}^{\text {th }}$ \\
\hline Alcator C-Mod & 2.7 & 0.67 & 2.7 & 21.1 & 1.66 & 1.7 & 1.4 \\
& 2.7 & 0.67 & 2.7 & 29.4 & 3.74 & 3.2 & 1.6 \\
& 2.7 & 0.67 & 3.8 & 36.8 & 4.75 & 1.2 & 1.3 \\
& 2.7 & 0.67 & 3.8 & 26.6 & 6.67 & 3.7 & 1.6 \\
& 2.7 & 0.67 & 5.4 & 21.6 & 2.78 & 1.9 & 1.1 \\
Compass & 2.7 & 0.67 & 5.4 & 23.2 & 4.77 & 2.3 & 1.2 \\
& 2.6 & 0.56 & 1.15 & 26.9 & 1.04 & 2.2 & 1.5 \\
JET & 4.2 & 0.56 & 1.15 & 20.2 & 0.68 & 2.0 & 2.5 \\
TCV & 3.0 & 2.96 & 2.53 & 29.6 & 0.26 & 3.9 & 2.5 \\
& 5.0 & 0.87 & 1.2 & 20.8 & 0.35 & 3.8 & 3.4 \\
Tore Supra & 5.0 & 0.87 & 1.2 & 31.5 & 0.39 & 3.8 & 3.1 \\
& 5.4 & 2.23 & 3.2 & 10 & 0.22 & 5.3 & 4.5 \\
& 9.4 & 2.23 & 3.85 & 14.0 & 0.14 & 7.0 & 6.1 \\
& 4.9 & 2.23 & 3.85 & 18.0 & 0.40 & 4.0 & 3.6 \\
\hline
\end{tabular}

95\% confidence interval obtained from the fitting of $L_{p}$. Ion temperature measurements were available only in Tore Supra discharges $\left(T_{\mathrm{i} 0} \approx 50 \mathrm{eV}, 57 \mathrm{eV}\right.$ and $57 \mathrm{eV}$ for the $q=5.4,9.4$, and 4.9 discharges, respectively). When $T_{\mathrm{i}}$ is not available we approximate $T_{\mathrm{i}}=T_{\mathrm{e}}$ and $L_{T_{\mathrm{i}}}=L_{T_{\mathrm{e}}}$. We note that the assumption $T_{\mathrm{i}}=T_{\mathrm{e}}$ is only expected to hold at high collisionality, while $L_{T_{\mathrm{e}}}$ and $L_{T_{\mathrm{i}}}$ are correlated, as discussed in [3].

We have also applied our scaling to the ITER start-up phase, using as indicative parameters $R=6.2 \mathrm{~m}, B=5.2 \mathrm{~T}$, $T_{\mathrm{e} 0}=50 \mathrm{eV}, n_{\mathrm{e} 0}=10^{19} \mathrm{~m}^{-3}$, and $q=5-8$, obtaining $L_{p}=6-10 \mathrm{~cm}$. We note that, as in the case of ITER, when $T_{\mathrm{e}}$ and $T_{\mathrm{i}}$ are unknown at the LCFS, it is possible to relate density and temperature in equation (4) to the power flux in the SOL, $P_{\text {in }}$. In order to do that, one can assume that $P_{\text {in }}$ is equal to the pressure loss at the limiter plate, integrated over the full SOL width, thus obtaining $P_{\text {in }} \sim n\left(T_{\mathrm{e}}+T_{\mathrm{i}}\right)^{3 / 2} r L_{p} / q$, under the hypothesis that $L_{p} \propto L_{n} \propto L_{T}$. Note, however, that Langmuir probe measurements have shown that SOL power balance is subject to a large uncertainty [7], and that the hypothesis $L_{p} \propto L_{n} \propto L_{T}$ is not always satisfied.

Finally, it is noted that recent analysis of reciprocating probe measurements show that sometimes very steep pressure gradients are found just outside the LCFS. This effect results in a very narrow (a few $\mathrm{mm}$ ) feature where most of the heat flux can be dissipated $[8,9]$, and it might lead to enhanced power loads near the apex of the ITER inner first wall. This phenomenon could involve plasma transport across the separatrix and neoclassical transport effects may be important [28]. In our current simulations, we have found that only one length scale was needed to describe the SOL profiles. The steepening of the SOL profiles near the LCFS will be addressed in the future by including a closed flux surface region within the non-linear simulations.

In conclusion, we have obtained a theory-based scaling for the SOL pressure gradient length in inner-wall limited discharges. The scaling, provided by equations (3) and (4), arises as a natural consequence of the balance between parallel losses at the plasma sheath and perpendicular turbulent transport driven by a resistive instability, with the gradient removal model used as a saturation mechanism. The scaling has a clear physical interpretation that clarifies the role of plasma current, machine size, and collisionality in determining the SOL width.

The predictions of our model have been compared in figure 1 against flux-driven non-linear turbulence simulations and in figure 2 against tokamak experimental data. The nonlinear simulations match the analytical model within a wide range of parameters, including a case where the dimensionless parameters match very closely the SOL of the TCV tokamak. An initial comparison with experimental data is also very encouraging, although agreement between the theory and experiment appears to deteriorate at the highest density Alcator C-Mod discharges, in particular at the lowest temperatures. It is possible that parallel temperature gradients become important in these discharges [20]—in this case it would be necessary to use a different estimate for the parallel losses in equation (1) and, in general, an important role in the SOL dynamics might be played by neutral particles.

It is remarked that the physical model used for $k_{\mathrm{b}}$ and $\gamma_{\mathrm{b}}$ was chosen for simplicity, as it is meant to capture the parallel dynamics of long wavelength RBMs in an analytically tractable way. A reduced model including all geometry effects, compressible flows, electron inertial effects, diamagnetic stabilizations, ion temperature, etc, could be developed using a linear stability code to compute $\gamma / k_{\theta}$. We anticipate, however, that the physical mechanism responsible for setting $L_{p}$ should be robust and, moreover, it provides a framework that could be extended to more sophisticated configurations where the instability driving turbulent transport could be different.

\section{Acknowledgments}

We acknowledge useful discussions with F. Capel, W.A. Cooper, J. Graves, T.-M. Tran, and S.J. Zweben. Part of the simulations presented herein were carried out at the Swiss National Supercomputing Centre (CSCS) under project ID s346; and part were carried out using the HELIOS supercomputer system at Computational Simulation Centre of 
International Fusion Energy Research Centre (IFERC-CSC), Aomori, Japan, under the Broader Approach collaboration between Euratom and Japan, implemented by Fusion for Energy and JAEA. This work was supported by the Swiss National Science Foundation, partially supported by GA CR P205/12/2327 and MSMT LM2011021, and by EURATOM, and carried out within the framework of the European Fusion Development Agreement. The views and opinions expressed herein do not necessarily reflect those of the European Commission.

\section{References}

[1] Zweben S.J., Scott B.D., Terry J.L., LaBombard B., Hughes J.W. and Stotler D.P. 2009 Comparison of scrape-off layer turbulence in Alcator C-Mod with three dimensional gyrofluid computations Phys. Plasmas 16082505

[2] Kočan M. and Gunn J.P. 2010 Comparison of scrape-off layer profiles in outboard-versus inboard-limited plasmas in Tore Supra Plasma Phys. Control. Fusion 52045010

[3] Kočan M. et al 2011 Measurements of ion energies in the tokamak plasma boundary Proc. 19th Int. Conf. on Plasma-Surface Interactions in Controlled Fusion (San Diego, CA); J. Nucl. Mater. 415 (Suppl. 1) S1133-8

[4] Fundamenski W. et al and JET-EFDA Contributors 2011 Multi-parameter scaling of divertor power load profiles in $\mathrm{D}, \mathrm{H}$ and $\mathrm{He}$ plasmas on JET and implications for ITER Nucl. Fusion $\mathbf{5 1} 083028$

[5] Eich T., Sieglin B., Scarabosio A., Fundamenski W., Goldston R.J. and Herrmann A. 2011 Inter-ELM power decay length for JET and ASDEX Upgrade: measurement and comparison with heuristic drift-based model Phys. Rev Lett. 107215001

[6] Makowski M.A. et al 2012 Analysis of a multi-machine database on divertor heat fluxes. Phys. Plasmas 19056122

[7] Gunn J. et al 2013 Scrape-off layer power flux measurements in the Tore Supra tokamak Proc. 20th Int. Conf. on Plasma-Surface Interactions in Controlled Fusion Devices (San Diego, CA); J. Nucl. Mater. 438 (Suppl. 0) S184-8

[8] Silva C., Arnoux G., Devaux S., Frigione D., Groth M., Horacek J., Lomas P.J., Marsen S., Matthews G. and Pitts R.A. 2013 Comparison of scrape-off layer transport in inner and outer wall limited JET plasmas Proc. 20th Int. Conf. on Plasma-Surface Interactions in Controlled Fusion Devices (Aachen); J. Nucl. Mater. 438 (Suppl.) S189-93

[9] Arnoux G. et al and the JET-EFDA Contributors 2013 Scrape-off layer properties of ITER-like limiter start-up plasmas in JET Nucl. Fusion 53073016

[10] Sarazin Y. et al 2003 Theoretical understanding of turbulent transport in the SOL J. Nucl. Mater. 313 796-803

[11] Garcia O., Horacek J., Pitts R., Nielsen A., Fundamenski W., Naulin V. and Rasmussen J.J. 2007 Fluctuations and transport in the TCV scrape-off layer Nucl. Fusion 47667

[12] Myra J.R. et al and the NSTX Team 2011 Reduced model simulations of the scrape-off-layer heat-flux width and comparison with experiment Phys. Plasmas 18012305

[13] Militello F., Naulin V. and Nielsen A.H. 2013 Numerical scalings of the decay lengths in the scrape-off layer Plasma Phys. Control. Fusion 55074010

[14] Ricci P., Halpern F.D., Jolliet S., Loizu J., Mosetto A., Fasoli A., Furno I. and Theiler C. 2012 Simulation of plasma turbulence in scrape-off layer conditions: the GBS code, simulation results and code validation Plasma Phys. Control. Fusion 54124047

[15] Ricci P. and Rogers B.N. 2013 Plasma turbulence in the scrape-off layer of tokamak devices Phys. Plasmas 20010702

[16] Halpern F.D., Jolliet S., Loizu J., Mosetto A. and Ricci P. 2013 Ideal ballooning modes in the tokamak scrape-off layer Phys. Plasmas 20052306

[17] Rogers B.N. and Dorland W. 2005 Noncurvature-driven modes in a transport barrier Phys. Plasmas 12062511

[18] Ricci P., Rogers B.N. and Brunner S. 2008 High- and low-confinement modes in simple magnetized toroidal plasmas Phys. Rev. Lett. 100225002

[19] Mosetto A., Halpern F.D., Jolliet S. and Ricci P. 2012 Low-frequency linear-mode regimes in the tokamak scrape-off layer Phys. Plasmas 19112103

[20] Stangeby P. 2000 The Plasma Boundary of Magnetic Fusion Devices (Bristol: Institute of Physics Publishing) pp 196-204

[21] Ribeiro T.T. and Scott B. 2008 Gyrofluid turbulence studies of the effect of the poloidal position of an axisymmetric debye sheath Plasma Phys. Control. Fusion 50055007

[22] Zeiler A., Biskamp D., Drake J.F. and Rogers B.N. 1998 Transition from resistive ballooning to $\eta_{\mathrm{i}}$ driven turbulence in tokamaks Phys. Plasmas 5 2654-63

[23] Mosetto A., Halpern F.D., Jolliet S., Loizu J. and Ricci P. 2013 Turbulent regimes in the tokamak scrape-off layer Phys. Plasmas 20092308

[24] Rogers B.N., Drake J.F. and Zeiler A. 1998 Phase space of tokamak edge turbulence, the $L-H$ transition, and the formation of the edge pedestal Phys. Rev. Lett. 81 4396-9

[25] LaBombard B., Hughes J., Mossessian D., Greenwald M., Lipschultz B., Terry J. and the Alcator C-Mod Team 2005 Evidence for electromagnetic fluid drift turbulence controlling the edge plasma state in the Alcator C-Mod tokamak Nucl. Fusion 451658

[26] Braginskii S.I. 1965 Transport Processes in a Plasma (Reviews of Plasma Physics vol 1) (New York: Consultants Bureau)

[27] Loizu J., Ricci P., Halpern F.D. and Jolliet S. 2012 Boundary conditions for plasma fluid models at the magnetic presheath entrance Phys. Plasmas 19122307

[28] Goldston R. 2012 Heuristic drift-based model of the power scrape-off width in low-gas-puff H-mode tokamaks $\mathrm{Nucl}$. Fusion 52013009 